\title{
Structural and dynamical analysis of integrated human/SARS-CoV-2 metabolic models present novel treatment strategies against COVID-19
}

Bridget P. Bannerman ( $\sim$ bee1bee22@gmail.com )

University of Cambridge https://orcid.org/0000-0002-5746-8283

Jorge Julvez

University of Zaragoza https://orcid.org/0000-0002-7093-228X

Alexandru Oarga

University of Zaragoza https://orcid.org/0000-0002-7271-733X

\section{Pablo Moreno}

European Molecular Biology Laboratory, European Bioinformatics Institute (EMBL-EBI), Cambridge https://orcid.org/0000-0002-9856-1679

\section{Tom L Blundell}

University of Cambridge https://orcid.org/0000-0002-2708-8992

\section{R. Andres Floto}

University of Cambridge

\section{Article}

Keywords: SARS-CoV-2, pathogenetic mechanism, cell function, human cells

Posted Date: January 15th, 2021

DOl: https://doi.org/10.21203/rs.3.rs-46892/v2

License: (c) (i) This work is licensed under a Creative Commons Attribution 4.0 International License.

Read Full License 


\section{Structural and dynamical analysis of integrated human/SARS-CoV-2 metabolic models present novel treatment strategies against Covid-19}

Bannerman, $\mathrm{BP}^{1^{*}}$, Julvez, $\mathrm{J}^{3}$, Oarga, $\mathrm{A}^{3}$, Moreno $\mathrm{P}^{4}$, Blundell, $\mathrm{TL}^{2}$, Floto, $\mathrm{RA}^{1}$

${ }^{1}$ Molecular Immunity Unit, Department of Medicine, University of Cambridge, UK

${ }^{2}$ Department of Biochemistry, University of Cambridge, UK

${ }^{3}$ University of Zaragoza, Spain

${ }^{4}$ EMBL-EBI. European Bioinformatics Institute, UK

The coronavirus disease 2019 (COVID-19) pandemic caused by the new coronavirus (SARSCoV-2) is currently responsible for over 500 thousand deaths in 216 countries across the world and is affecting over 10 million people. The absence of FDA approved drugs against the new SARS-CoV-2 virus has highlighted an urgent need to design new drugs. We developed an integrated model of the human cell and the SARS-CoV-2 virus to provide insight into the pathogenetic mechanism of the virus and to support current therapeutic strategies. We show the biochemical reactions required for the growth and general maintenance of the human cell, first of all, in its healthy state. We then demonstrate how the entry of the SARS-CoV-2 virus into the human cell causes biochemical and structural changes, leading to a change of cell functions or cell death. We have completed a comparative analysis of our model and other previously generated cell type models and highlight 48 pathways and over 800 reactions hijacked by the virus for its replication and survival. We designed a new tool which predicts 15 unique reactions as drug targets from our models (the integrated human macrophage, human airway epithelial cells and the SARS-CoV-2 virus) and provide a platform for future studies on viral entry inhibition and drug optimisation strategies.

\section{INTRODUCTION}

SARS-COV-2, the causative agent of the COVID-19 disease, belongs to a group of viruses commonly known as $\beta$-coronavirus. This class of viruses is responsible for mild-to-fatal respiratory tract infections in animals and birds. Whilst the common cold is more commonly associated with the mild forms of the disease, the previous MERS and SARS-2002 infections and the current COVID-19 disease belong to the group of fatal diseases. The genome of the virus responsible for the ongoing COVID-19 disease, SARS-CoV-2, has $\sim 80 \%$ sequence identity to SARS-CoV and $96 \%$ identical at the whole-genome level to a bat coronavirus (Zhou et al., 2020). The SARS-CoV-2 virus affects the lower respiratory tract cells and the upper cells in the pharyngeal region (Huang et al., 2020; Chen et al., 2020); and the range of viral infections ranges from asymptomatic, mild, moderate and severe cases. Previous studies in China show that $86 \%$ of cases of infection and the contagiousness of the virus were undocumented before travel restrictions were imposed ( $\mathrm{Li}$ et al., 2020). Therefore, there are still many unknown factors regarding the stages of infection and transmissibility patterns of the virus. Studies in France demonstrate the transmission potential of asymptomatic persons and suggest varying dynamics of transmission in children (Danis et al., 2020). The human 
angiotensin-converting enzyme 2 (human-ACE-2 protein) has been identified as the cell receptor for both the SARS-2002 virus and the SARS-CoV-2 virus. The ACE-2 enzyme, which has a primary function of controlling blood pressure, is usually found in the epithelial cells of the heart, lungs, kidneys and intestine (Hamming et al., 2014; Donoghue et al., 2000).

The mechanism of replication of the SARS-CoV-2 virus in the human cell involves an initial binding and attachment of the spike (S) glycoprotein to the ACE2 receptor of its host. During endocytosis, the genetic material of the virus is injected into the host cell, where it loses its protective envelope. The virus, now ready for replication, is released into the nucleus of the human cell (Fig 1). Subsequent assembly and maturation of viral proteins lead to cell death and a proliferation of the virus within the human body.

The lack of FDA approved drugs against Covid-19, coupled with the difficulties encountered globally in containing the virus, prompted the WHO to declare the outbreak a pandemic in March 2020. The has led to intensified efforts around the world to fight this disease. Previous studies in drug target identification against viral diseases such as Zika, Chikungunya and Dengue by Aller et al., 2018 introduced a system of integrating the host's macrophage and viral metabolic networks to predict a set of host reactions which, when constrained, can inhibit viral production. A recent study by Renz et al., 2020 demonstrates a similar approach and predicts drug targets against the SARS-CoV2 virus. Targets of known antiviral drugs predicted from both studies demonstrate the applicability of the integrated human/virus metabolic modelling in drug target identification.

We have built on these approaches by developing an integrated epithelial cell / SARS-CoV-2 virus metabolic model and employed a combination of structural and dynamical analyses to assess the model and make predictions. We have designed and developed a new tool (findCPcli) to carry out such analyses and to predict drug targets. We have also performed a comparative analysis of our model and another previously generated cell-type models.

\section{RESULTS}

\section{Comparative analysis of integrated models of infected human epithelial cell and the macrophage cell with the SARS-CoV-2 virus}

We constructed an integrated genome-scale metabolic model (GEM) of the human airway epithelial cell (Wang et al., 2017), with the SARS-CoV-2 virus using the methods described in Aller et al., 2018 and Renz et al., 2020. The new GEM (iBBEC4660) was refined by using the human metabolic networks in the HumanCyc database (Romero et al., 2004). We performed a comparative analysis of the essential and unique reactions needed for the viability of the virus in the epithelial cell/ SARS-CoV-2 integrated model and the GEM constructed by Renz et al., 2020. Our results show how the virus heightens its virulence mechanisms by modifying 
the host's defences within different cell compartments. Consequently, we suggest treatment regimens based on different stages of viral infection and replication.

\section{Host dependent metabolic pathways}

We initially demonstrated the biochemical requirements for the growth and maintenance of the human airway epithelial and macrophage cells and used the integrated models to show the essential host reactions needed for the survival and viability of the SARS-CoV-2 virus within the host's cell compartments. We have validated our models by mapping the experimentally characterised human/SARS-CoV-2 virus protein-protein interaction data from Gordon et al., 2020 on the in-silico virus-integrated human macrophage and epithelial cells. We identified 48 metabolic pathways from 334 metabolic pathways in the human metabolic network, including the biosynthesis and degradation pathways of amino acids, fatty acids, carbohydrates, amines, cofactors as well as core components of the central mRNA metabolism (Fig 2).

The 48 metabolic pathways that were mapped to the protein-protein interaction network produced by Gordon et al., 2020 are referred to as PPi-Pathway Intersection nodes in this manuscript (Fig 3). These include cysteine, methionine and selenocysteine amino acid biosynthetic pathways, C20 prostanoid hormone biosynthetic pathways, Vitamin D3 and Vitamin $\mathrm{K}$ epoxide cycle. The degradation pathways identified include the lysine, tryptophan, methionine, fatty acid degradation, ceramide and sphingolipid recycling pathways and phospholipases degradation; amine and heme degradation (Fig 3).

Our results identify host dependency factors required for the SARS-CoV-2 virus infection, replication, survival and viability within different cell compartments and provide insight into novel treatment strategies.

\section{Essential reactions for the host and viral metabolism}

The Flux Balance Analysis (FBA) algorithm (Orth et al., 2010) was used to compute both the maximum growth rate of the cell in the absence of virus and the maximum growth rate of the virus in the cell (host optimum and virus optimum conditions). We identified 52 essential reactions in the macrophage ( $\mathrm{A} A \mathrm{~B}-\mathrm{AM} \varnothing-1410$ ) model and 10 reactions in the epithelial cell model (iBBEC4660) essential for the virus to propagate (Tables 1 in S1 Table and 2 in S2 Table). It was also demonstrated that: a) the maximum growth rate of the macrophage cell in the absence of virus was $0.0269 \mathrm{~h}^{-1}$ (Table 1 in S1 Table); and 0.012 for the human airway epithelial cell (Table 2 in S2 Table); b) the maximum growth rate of the virus in the macrophage cell was $0.0144 \mathrm{~h}^{-1}$ and 0.0181 in the human airway epithelial cell. These numerical results mean that $0.0144 \mathrm{~h}^{-1}$ is the theoretical maximum of the growth rate of the virus in the human macrophage cell. If this flux is assigned to the viral growth reaction, then Flux Variability 
Analysis (FVA) (Orth et al., 2010) can be used to calculate the ranges of fluxes allowed for the remaining reactions in the cell while the virus is being replicated at its optimum condition. The execution of FVA under such conditions produced a zero growth of the host cell, i.e. both the lower and upper flux bounds of the reaction indicate that the growth is zero. This means that if the virus is replicating at its maximum rate then the cell cannot reproduce.

\section{Bottleneck reactions and the prioritization of potential drug targets}

The bottleneck reactions identified by the findCPcli tool are unique reactions in a metabolic network required for the growth and survival of the organism and, like chokepoint reactions, are potential drug targets ( Yeh et al., 2004; Oarga et al., 2020). Whilst the classical chokepoint reactions identify unique reactions from a stoichiometric model, we improve on this approach by using the structural and dynamical information of the integrated Human/SARS-CoV-2 metabolic model within the airway epithelial cell and the macrophage cell to predict potential drug targets against the SARS-CoV-2 virus.

We initially identified 1595 bottleneck reactions required for the virus' maintenance and replication in the human macrophage cell; these include pathways in lipid metabolism, coenzyme transport and metabolism, energy production and conversion, amino acid and nucleotide transport and metabolism (Table 1 in S1 Table). In the human airway epithelial cell, 1819 bottleneck reactions were initially identified; these include the biosynthesis and degradation pathways of amino acids, fatty acids, carbohydrates, amines, cofactors as well as some components of the central mRNA metabolism (Table 2 in S2 Table).

To validate/account for the results, and because each bottleneck reaction should be balanced by at least one other reaction that produces or consumes that metabolite, we have excluded reactions in the model with dead-end metabolites. The bottleneck reactions are further prioritised by interrogating the dynamical information in the model using the flux variability analysis, which determines if a reaction is reversible. The bottleneck reactions are potential drug targets as they are indispensable for the maintenance and replication of the virus within the host. In order to rank the potential drug targets identified, we prioritised enzymes for unique reactions that occur at the nodes of intersection between the bottleneck and essential reactions and the experimental results from the human/virus protein-protein interaction network (Gordon et al., 2020) (Fig 3). We refer to these as PPi-Pathway intersection nodes.

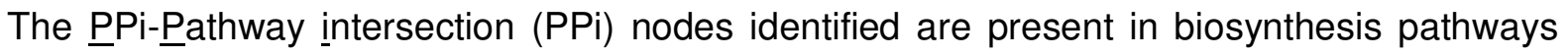
such as the cysteine and S-adenosyl-L-methionine biosynthetic pathways. In both pathways, the enzyme S-adenosylmethionine synthase (Mat2b), catalyses the phosphorylation reaction of methionine to S-adenosyl-L-methionine. During infection, the viral protein Nsp9 is seen to react with MAT2B (Gordon et al., 2020) (Fig 4a/b). Another viral protein, Nsp8 also interacts 
with the enzyme O-phosphoseryl-tRNA(Sec) selenium transferase (SEPSECS), which catalyses the last step of the L-selenocysteine biosynthesis pathway (Fig 5).

PPi nodes also occur in a network of various fatty acid and stearate biosynthetic pathways with Nsp2 interacting with the very long-chain acyl-CoA synthetase (SLC27A2) (Fig 6a). In other fatty acid biosynthetic pathways, $Y$-linolenate biosynthesis, Nsp7 interacts with ACSL3 (Fig 6a)The viral protein, Nsp2, also interacts with POR in other pathways including vitamin D3 biosynthesis, L-tryptophan degradation, ceramide and sphingolipid recycling (Fig 6b).

In carbohydrate metabolism, a PPi node is identified at the glycan \& oligosaccharide biosynthetic pathways, and specifically where two mannose residues are added in $\alpha(1 \rightarrow 2)$ linkages to the nascent oligosaccharide and catalysed by the enzyme ALG11. The viral protein Nsp4 interacts with ALG11 during the infection of the SARS-CoV-2 virus (Fig 7). Other viral proteins, Nsp7 (Fig 8) reacts with ACSL3 and ORF8a interacts with HS2ST1 (Fig 8b), a key enzyme involved in the heparan sulfate biosynthesis pathway. The first enzyme of the $\mathrm{N}$-linked oligosaccharide processing pathway, mannosyl-oligosaccharide $\alpha$-1,2-glucosidase (MOGS), also interacts with Nsp7 and ORF8a (Fig 8c).

PPi nodes specific to the human macrophage cell include the O-phosphoseryl-tRNA(Sec) selenium transferase in the L-selenocysteine biosynthetic pathway, which interacts with the viral protein Nsp8. The alkylglycerone-phosphate synthase/Nps7 PPi node, which is present in the Phospholipid/Plasmalogen biosynthetic pathway is also specific to the macrophage cell. Alternatively, PP-pathway intersection nodes common to both human airway epithelial cell and the macrophage cell are the MAT2B/Nsp9 intersection pathways present in the cysteine metabolism and L-methionine degradation. We did not identify PPi nodes specific to the human epithelial cell.

\section{Discussion}

Metabolic pathway perturbations in the human cell due to the Covid-19 disease are a reflection of the viral entry and infection of SARS-CoV-2 and of the immune regulation changes in the human body. We have used in silico system models to study the interactions of SARS-CoV-2 in the host and propose new treatment management regimens. We have built on studies using the human alveolar macrophage model iAB-AMØ-1410 (Bordbar et al., 2010) as host cells and SARS-CoV-2 (Renz et al., 2020); influenza (Aller et al. 2017), and tuberculosis as pathogens. Previous studies have also demonstrated the role of the angiotensin converting enzyme 2 (ACE2) as the receptor for both the SARS-CoV and the SARS-CoV-2. The ACE2 cells are expressed in the human airway epithelial cells (Yan et al., 2020; Jia et al., 2005). In this study, we have constructed an integrated human epithelial cell and the SARS-CoV-2 virus to provide insight into the infection patterns of the virus in the human body. 
Our in silico comparative analyses of the SARS-CoV-2 viral infection between two different conditions (infected human macrophage and airway epithelial cells) show the requirements of viability of the virus between these two conditions. Our results compliment previous efforts to propose drug targets and repurposing strategies including the SARS-CoV-2-Human ProteinProtein Interaction Map (Gordon et al., 2020) and studies from Joseph Steward, 2020 which identified host dependency factors facilitating virus infection. We also provide additional resource to the Covid-19 disease map (Ostaszewsli et al., 2020). In addition, we have designed a new algorithm (Oarga et al., 2020) and used the dynamic information of the human/virus models to predict new treatment regimens. Here we demonstrate the flux changes from lipid metabolism, cofactor biosynthetic pathways, redox balance and immune regulation indicative of pathogenic reactions arising from the Covid-19 viral infections.

\section{Inhibition of viral entry and replication}

The components of the plasma membrane, such as cholesterol and sphingolipid-rich lipid, are involved in virus penetration, entry, replication and infection (Wang et al., 2008; Abu-Farha et al., 2020). In this study, we demonstrate current therapeutic strategies that interfere with different stages of the viral cycle by targeting lipid metabolism as well as proposing new treatment strategies.

The dynamical changes of flux metabolism in our in-silico virus optimal models show significant increase in viral infection. Four reactions involved in the biosynthesis of fatty acids with predicted non-zero fluxes in the host model exhibit an average increase of $190 \%$ in their maximum fluxes in the viral model (the maximum increase is $298 \%$ ). The average increase of 32 reactions in lipid metabolism with non-zero fluxes in the host model is $277 \%$ (the maximum increase is $498 \%$ ). With respect to sphingolipid metabolism, 14 out of 15 reactions with non-zero fluxes in the host model exhibit an average increase of $228 \%$ (the maximum increase is 298\%) and similar increases in phospholipases and palmitic acid biosynthesis (Fig 6). We show an average increase of $190 \%$ in cholesterol and fatty acid metabolism during viral infection and demonstrate the essentiality of these pathways to SARSCoV-2 virus. Previous studies have shown that cholesterol and fatty acids are main components of the viral membranes and needed for viral replication (Heaton and Randall, 2011); therefore, drugs inhibiting these pathways such as AM580, Statins, Fibrate (Fiévet et al., 2009) will be essential for both early and late stages of the Covid-19 disease.

Sphingolipids are composed of both hydrophobic and hydrophilic units and play a large role in the endocytic or exocytic viral entry processes into the cell (Dimitrov et al., 2004). The pHdependent endocytic process is further enhanced by the presence of clathrin, a protein present in the plasma membrane, Golgi apparatus and in the cytoplasm, whilst the exocytic 
route involves viral crossing through the plasma membrane at neutral $\mathrm{pH}$. Our results show a 3-fold increase in sphingolipid metabolism during viral infection (Fig 6); we hypothesise that drugs inhibiting sphingolipid metabolism and/or the endocytosis process will inhibit infection of SARs-CoV-2 virus. Indeed, a sphingosine kinase-2 (SphK2) inhibitor, Opaganib, which has proved beneficial in the treatment of Covid-19 is currently in phase 2/3 clinical trials in the US, and now in UK and Italy. Previous studies also demonstrate that chloroquine and hydroxychloroquine elevate the $\mathrm{pH}$ of endosomes in the cells and directly inhibits endocytosis and the exocytic process (Munro et al., 1997, Devaux et al., 2020). More recent studies have also shown that artemisinin-inhibited endocytosis (Uzzun et al., 2020, Hoppe et al., 2004). A recent study by Abu-Farha described other lipid modifying drugs including LJ-001, Arbidol, Methyl-B-cyclodextrin (Mazzon and Marsh 2019; de Wilde et al., 2014; Blaising et al., 2013. We highlight critical reactions as drug targets for lipid metabolism for the SARS-CoV-2 virus (table 2) and in our PPi-pathway intersection nodes (Fig 3).

Our lists of bottleneck, essential reactions and PPi-pathway intersection nodes also include critical points in the biosynthesis of phospholipids. We show that these reactions are essential for viral infection and replication (Fig 3) and propose that targeting the phospholipase enzyme or the interacting Nsp2 protein could inhibit viral replication. Our results support previous studies from Muller et al., 2018, that targeting the phospholipase enzyme could inhibit the early stage of Covid-19 disease.

\section{Redox homeostasis and antioxidant therapy}

Redox homeostasis refers to the ability of the cell to maintain its balance amidst infections and other unstable cellular environmental factors. Delgardo-Roche and Mesta, 2020 have described oxidative stress as a key player in Severe Acute Respiratory Syndrome Coronavirus (SARS-CoV) Infection with cytokine production. Foyer and Noctor 2005 have previously shown that antioxidants, such as glutathione and ascorbate, are important metabolites for the cellular redox state. Our studies have identified key target enzymes involved in the metabolism of glutathione and ascorbic acid as bottleneck and essential reactions; including glutathione synthase, glutathione peroxidase and ascorbic acid oxidase (Table 2). We also demonstrate an increase in flux of these enzymatic reactions on infection of the virus. In a recent study, Horowitz et al., 2020 previously demonstrated how the use of high dose oral and/or IV glutathione on severe outcomes of SARS-CoV-2 virus led to favourable treatment outcomes. Other studies have shown that steroids such as dexamethasone and Methylpredisone to treat severe cases of Covid-19. Due to possible side effects of steroid treatment, we propose the use glutathione as therapy for severe cases of Covid-19 in the aged population and other severe cases with cytokine storm syndrome. 


\section{Immune regulation}

The SARS-CoV-2 virus is able to proliferate unhindered in infected cells, due to the lack of immunity in humans (Felsenstein et al., 2020). The result is cell death, a release of viral particles to the extracellular environment and a general hyperactivity of the immune system in some patients with severe Covid-19 disease and subsequent, lung inflammation and cytokine syndrome. Immunocompromised patients or those with underlying symptoms such as diabetes, hypertension and transplantation are most affected (Zhong et al., 2020). Whilst clinical trials are ongoing worldwide with various antivirals and immune modulating treatments, there is currently limited knowledge on the host dependency factors responsible for the individual outcomes of the disease. Our results provide insight into the immune evasion strategies of SARS-CoV2; we demonstrate changes in the flux metabolism of vitamin D and tryptophan metabolism during viral infection. Vitamin $D$ is important for bone growth and turnover and a low vitamin D status is associated with an increased susceptibility to upper respiratory tract infections (Mitchell F, 2020). Previous studies have shown that a supplementation of vitamin D prevents acute respiratory tract infections (Martineau et al., 2016). Our results highlight vitamin D as an essential reaction in the PPi-pathway intersection nodes and we show the viral protein Nsp2 interaction with key enzymes in the vitamins $D$ and C metabolism pathways (Fig 3 and 6). SARS-CoV-2 viral infection causes metabolic perturbations of vitamin $\mathrm{D}$ metabolism in the host resulting to disruptions in cellular homeostasis. We propose support therapy management strategies where vitamin $D$ supplements are provided to all Covid-19 patients. Our results also show that tryptophan, melatonin and prostaglandins, important compounds for immunity and homeostasis (Platten et al., 2019; Gitto et al., 2010), are affected by the infection of SARS-CoV-2 virus and we provide insight into the viral mechanism of action within the human body.

In summary, we have provided a platform for drug target prediction against Covid-19, and for future studies on viral entry inhibition, antioxidant therapy and immune regulation.

\section{METHODS}

We manually curated the human airway epithelial cell initially constructed by Wang et al., 2017 with gene expression datasets of the human airway epithelial cell (Deprez et al., 2020; Braga et al., 2020) and the humancyc database (Romero et al., 2014) to produce a new GEM, (iBBEC4660); i for in-silico, BB for the first author's name, EC for airway epithelial cell and 4660 for the number of open reading frames. In order to assess and predict the performance of the models, we made use of Flux Balance Analysis (FBA) and Flux Variability Analysis (FVA) (Orth et al., 2010). FBA is a computational method that can be applied efficiently to genome scale models to estimate the fluxes of reactions at steady state. It is based on the solution of a linear programming problem that maximizes an objective function of interest subject to a set of constraints on the fluxes of the reactions. The linear programming problem associated with FBA can be expressed as: 


$$
\begin{aligned}
& \max c \cdot v \\
& \text { s.t. } S \cdot v=0 \\
& L \leq v \leq U
\end{aligned}
$$

where $v$ is the vector of fluxes, $c$ represents the objective, $S$ is the stoichiometry matrix, and $L$ and $U$ are lower and upper bounds on the fluxes. Thus, $c \cdot v$ is the objective function, which usually refers to the growth rate of the organism, and $S \cdot v=0$ represents the balance of fluxes at steady state.

FVA is also based on the solution of linear programming problems, and its main use is the computation of ranges of fluxes that are compatible with given flux constraints. For instance, if the growth rate predicted by FBA is $\mu_{\max }$, then the range of fluxes of a given reaction $i$ that are compatible with such growth rate can be obtained by minimizing and maximizing the following programming problem:

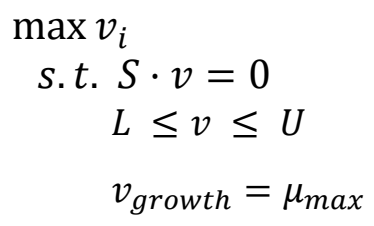

where $v_{\text {growth }}$ is the flux of the reaction associated with growth. FBA and FVA were applied on the metabolic network of the host, both with and without reaction modelling the production of the virus, by using the Python toolbox COBRApy (Ebrahim et al., 2013).

Bottleneck reactions, like chokepoint reactions, are required for the reaction synthesis and the removal of these reactions will cause an accumulation or depletion of the metabolites; they represent potential drug targets. The tool findCPCli is a command line application intended for the computation of bottleneck reactions on genome scale models. The source code of the tool is available at github.com/findCP/findCPcli. This tool is distributed as a Python package and requires an installation of a Python language interpreter with a version 3.5 or higher and the application can be installed with the pip package management tool.

The pathway maps were created with the pathway collage software (Paley et al., 2016) and the model was deposited in the BioModels database (Chelliah et al., 2015) and assigned the identifier "MODEL2007210001".

\section{ACKNOWLEDGEMENTS}

Authors of this manuscript are supported by The Wellcome Trust, The Cystic Fibrosis Trust and the Spanish Ministry of Science, Innovation and Universities. The authors would like to thank Jo Chukualim for the graphics and illustrations.

\section{AUTHOR CONTRIBUTIONS}

Conceived and designed the study: BP. Bannerman, RA. Floto. Acquisition and analysis of data: BP. Bannerman, J Julvez, A. Oarga. Data Interpretation: BP. Bannerman, RA. Floto, J Julvez, TL. Blundell, P. Moreno. Wrote the paper: B. P. Bannerman, J Julvez, A. Oarga, RA Floto. Revised the paper: B. P. Bannerman, J Julvez, A. Oarga, P. Moreno, TLBlundell, RAFloto 


\section{REFERENCES}

Abdul Alim Al-Bari. 2016. "Targeting Endosomal Acidification by Chloroquine Analogs as a Promising Strategy for the Treatment of Emerging Viral Diseases."

Abu-Farha, Mohamed, Alphonse Thanaraj, Mohammad G. Qaddoumi, Anwar Hashem, Jehad Abubaker, and Fahd Al-Mulla. 2020. "The Role of Lipid Metabolism in COVID-19 Virus Infection and as a Drug Target." Int. J. Mol. Sci 21 (10).

Aller, S, A Scott, M Sarkar-Tyson, and OS Soyer. 2018. "Integrated Human-Virus Metabolic Stoichiometric Modelling Predicts Host-Based Antiviral Targets against Chikungunya, Dengue and Zika Viruses." J. R. Soc. Interface 15: 20180125.

Blaising, Julie, Pierre L. Lévy, Stephen J. Polyak, Megan Stanifer, Steeve Boulant, and EveIsabelle Pécheur. 2013. "Arbidol Inhibits Viral Entry by Interfering with Clathrin-Dependent Trafficking." Antiviral Research 100 (1): 215-19.

Chelliah V et al. BioModels: ten-year anniversary. Nucl. Acids Res. 2015, 43(Database issue):D542-8

Chen, Y, Q Liu, and D Guo. 2020. "Emerging Coronaviruses: Genome Structure, Replication, and Pathogenesis." J. Med. Virol.

Danis, Kostas, Olivier Epaulard, Thomas Bénet, Alexandre Gaymard, Séphora Campoy, Elisabeth Bothelo-Nevers, Maude Bouscambert-Duchamp, et al. 2020. "Cluster of Coronavirus Disease 2019 (Covid-19) in the French Alps, 2020." Clinical Infectious Diseases, no. ciaa424 (April). https://doi.org/10.1093/cid/ciaa424.

Delgado-Roche, Livan, and Fernando Mesta. 2020. "Oxidative Stress as Key Player in Severe Acute Respiratory Syndrome Coronavirus (SARS-CoV) Infection." Archives of Medical Research 51 (5): 384-87. https://doi.org/10.1016/j.arcmed.2020.04.019.

Deprez, Marie, Laure-Emmanuelle Zaragosi, Marin Truchi, Sandra Ruiz Garcia, Marie-Jeanne Arguel, Kevin Lebrigand, Agnès Paquet, et al. 2019. "A Single-Cell Atlas of the Human Healthy Airways." BioRxiv, January, 2019.12.21.884759.

Devaux, Christian A, Jean-Marc Rolain, Philippe Colson, and Didier Raoult. 2020. "New Insights on the Antiviral Effects of Chloroquine against Coronavirus: What to Expect for COVID-19?" International Journal of Antimicrobial Agents 55 (5): 105938-105938. https://doi.org/10.1016/.ijantimicag.2020.105938.

Dimitrov, Dimiter S. 2004. "Virus Entry: Molecular Mechanisms and Biomedical Applications." Nature Reviews Microbiology 2 (2): 109-22. https://doi.org/10.1038/nrmicro817.

Donoghue, M, F Hsieh, E Baronas, K Godbout, K Robinson, R Jeyaseelan, RE Breitbart, and S Acton. 2000. "A Novel Angiotensin-Converting Enzyme-Related Carboxypeptidase (ACE2) Converts Angiotensin I to Angiotensin 1-9"." Circulation Research. 87 (5): e1-e9. https://doi.org/doi:10.1161/01.RES.87.5.e1.

Ebrahim, Ali, Joshua A Lerman, B Ø Palsson, and Daniel R Hyduke. 2013. "COBRApy: COnstraints-Based Reconstruction and Analysis for Python." BMC Systems Biology 7: 74.

Felsenstein, Susanna, Jenny A. Herbert, Paul S. McNamara, and Christian M. Hedrich. 2020. "COVID-19: Immunology and Treatment Options." Clinical Immunology 215 (June): 108448. https://doi.org/10.1016/j.clim.2020.108448.

Fiévet, Catherine, and Bart Staels. 2009. "Combination Therapy of Statins and Fibrates in the Management of Cardiovascular Risk." Current Opinion in Lipidology 20 (6): 505-11. https://doi.org/10.1097/MOL.0b013e328332e9ef.

Foyer, CH, and G Noctor. 2005. "Redox Homeostasis and Antioxidant Signaling: A Metabolic Interface between Stress Perception and Physiological Responses.Doi:10.1105/Tpc.105.033589." Plant Cell. 17 (7): 1866-75.

Gautret, Philippe, Jean-Christophe Lagier, and Philippe Parola. n.d. "Hydroxychloroquine and Azithromycin as a Treatment of COVID-19: Results of an Open-Label Non-Randomized Clinical Trial. Van Thuan Hoang, Line Meddeb , Morgane Mailhe , Barbara Doudier , Johan Courjon, Vale'rie Giordanengo, Vera Esteves Vieira, Herve' Tissot Dupont, Ste phane Honore', Philippe Colson, Eric Chabrie're , Bernard La Scola , Jean-Marc Rolain , Philippe Brouqui , Didier Raoult." https://doi.org/10.1016/j.ijantimicag.2020.105949.

Gitto, E, S Aversa, RJ Reiter, I Barberi, and S Pellegrino. 2011. "Update on the Use of Melatonin in Pediatrics." Journal of Pineal Research., no. 50: 21-28. 
Gordon, D. 2020. "A SARS-CoV-2-Human Protein-Protein Interaction Map Reveals Drug Targets and Potential Drug Repurposing." BioRxiv. https://doi.org/10.1101/2020.03.22.002386.

Hamming, I, W Timmens, Bulthius ML, AT Lely, G Navis, and H van Goor. 2014. "Tissue Distribution of ACE2 Protein, the Functional Receptor for SARS Coronavirus. A First Step in Understanding SARS Pathogenesis." The Journal of Pathology. 203 (2): 631-7. https://doi.org/10.1002/path.1570.

Heaton, Nicholas S., and Glenn Randall. 2011. "Multifaceted Roles for Lipids in Viral Infection." Trends in Microbiology 19 (7): 368-75. https://doi.org/10.1016/..tim.2011.03.007.

Hoffman. 2020. "SARS-CoV-2 Cell Entry Depends on ACE2 and TMPRSS2 and Is Blocked by a Clinically Proven Protease Inhibitor."

Hoppe, HC, DA van Schalkwyk, UI Wiehart, SA Meredith, J Egan, and BW Weber. 2004. "Antimalarial Quinolines and Artemisinin Inhibit Endocytosis in Plasmodium Falciparum." Antimicrob Agents Chemother 48 (7): 2370-78.

Horowitz, Richard I., Phyllis R. Freeman, and James Bruzzese. 2020. "Efficacy of Glutathione Therapy in Relieving Dyspnea Associated with COVID-19 Pneumonia: A Report of 2 Cases." Respiratory Medicine Case Reports 30 (January): 101063. https://doi.org/10.1016/i.rmcr.2020.101063.

Huang, C, and et al. 2020. "Clinical Features of Patients Infected with 2019 Novel Coronavirus in Wuhan, China." Lancet.

Huaxia. 'Favipiravir Shows Good Clinical Efficacy in Treating COVID-19: Official.' Xinhuanet.Com, 17 March 2020." n.d.

Jia, Hong Peng, Dwight C. Look, Lei Shi, Melissa Hickey, Lecia Pewe, Jason Netland, Michael Farzan, Christine Wohlford-Lenane, Stanley Perlman, and Paul B. McCray. 2005. "ACE2 Receptor Expression and Severe Acute Respiratory Syndrome Coronavirus Infection Depend on Differentiation of Human Airway Epithelia." Journal of Virology 79 (23): 14614. https://doi.org/10.1128/JVI.79.23.14614-14621.2005.

Li. 2005. "Structure of SARS Coronavirus Spike Receptor-Binding Domain Complexed with Receptor Fang Li1, Wenhui Li3, Michael Farzan3, Stephen C. Harrison1,2,* See All Authors and Affiliations Science 16 Sep 2005:" Science.

Li G, De Clercq E. Therapeutic Options for the 2019 Novel Coronavirus (2019-NCoV). Nature Reviews Drug Discovery 2020 Feb Doi:10.1038/D41573-020-00016-0." n.d.

Li, Ruiyun, Sen Pei, Bin Chen, Yimeng Song, Tao Zhang, Wan Yang, and Jeffrey Shaman. 2020. "Substantial Undocumented Infection Facilitates the Rapid Dissemination of Novel Coronavirus (SARS-CoV2)." Science, March, eabb3221. https://doi.org/10.1126/science.abb3221.

Li, Y, G Liu, J Zhang, X Zhong, and Z He. 2018. "Identification of Key Genes in Human Airway Epithelial Cells in Response to Respiratory Pathogens Using Microarray Analysis." BMC Microbiology 18 (58).

Li, Yinghua, Guangnan Liu, Jianquan Zhang, Xiaoning Zhong, and Zhiyi He. 2018. "Identification of Key Genes in Human Airway Epithelial Cells in Response to Respiratory Pathogens Using Microarray Analysis." BMC Microbiology 18 (1): 58. https://doi.org/10.1186/s12866-0181187-7.

Luo H1,2, Tang QL3, Shang YX2,3, Liang SB2,3, Yang M2,3, Robinson N2,4, Liu JP5,6." n.d.

Martineau, Adrian R, David A Jolliffe, Richard L Hooper, Lauren Greenberg, John F Aloia, Peter Bergman, Gal Dubnov-Raz, et al. 2017. "Vitamin D Supplementation to Prevent Acute Respiratory Tract Infections: Systematic Review and Meta-Analysis of Individual Participant Data." BMJ 356: i6583. https://doi.org/10.1136/bmj.i6583.

Mazzon, M, and M Marsh. 2019. "Targeting Viral Entry as a Strategy for Broad-Spectrum Antivirals (Https://Doi.Org/10.12688/F1000research.19694.1)." F1000Research 8 (F1000 Faculty Rev): 1628.

Menachery, V.D., Dinnon, K.H., III, Yount, B.L., Jr., McAnarney, E.T., Gralinski, L.E., Hale, A., Graham, R.L., Scobey, T., Anthony, S.J., Wang, L., et al. (2020). Trypsin Treatment Unlocks Barrier for Zoonotic Bat Coronaviruses Infection. J. Virol. 94 Https://Doi.Org/10.1128/JVI.01774-19." n.d.

Mitchell, Fiona. 2020. "Vitamin-D and COVID-19: Do Deficient Risk a Poorer Outcome?" The Lancet Diabetes \& Endocrinology 8 (7): 570. https://doi.org/10.1016/S2213-8587(20)301832.

Müller, Christin, Martin Hardt, Dominik Schwudke, Benjamin W. Neuman, Stephan Pleschka, and John Ziebuhr. 2018. "Inhibition of Cytosolic Phospholipase $A_{2} \alpha$ Impairs an Early Step of 
Coronavirus Replication in Cell Culture." Edited by Tom Gallagher. Journal of Virology 92 (4): e01463-17. https://doi.org/10.1128/JVI.01463-17.

Munro, R, E Morrison, A G McDonald, J A Hunter, R Madhok, and H A Capell. 1997. "Effect of

Disease Modifying Agents on the Lipid Profiles of Patients with Rheumatoid Arthritis." Annals of the Rheumatic Diseases 56 (6): 374. https://doi.org/10.1136/ard.56.6.374.

NHK World News 'China: Avigan Effective in Tackling Coronavirus.'” n.d.

Orth, JD, I Thiele, and B Ø Palsson. 2010. "What Is Flux Balance Analysis?" Nat Biotechnol 28 (3): 245-248.

Paley S, O'Maille PE, Weaver D, Karp PD. Pathway collages: personalized multi-pathway diagrams. BMC Bioinformatics. 2016;17(1):529. Published 2016 Dec 13. doi:10.1186/s12859-016-1382-1

Platten, Michael, Ellen A. A. Nollen, Ute F. Röhrig, Francesca Fallarino, and Christiane A. Opitz. 2019. "Tryptophan Metabolism as a Common Therapeutic Target in Cancer, Neurodegeneration and Beyond." Nature Reviews Drug Discovery 18 (5): 379-401. https://doi.org/10.1038/s41573-019-0016-5.

Renz, A, L Widerspick, and A Dra"ger. n.d. "FBA Reveals Guanylate Kinase as Potential Target for Antiviral Therapies against Coronavirus SARS-CoV-2." Https://Zenodo.Org/Record/3752641\#.XuiRQmpKg0o.

Romero, Pedro, Jonathan Wagg, Michelle L. Green, Dale Kaiser, Markus Krummenacker, and Peter D. Karp. 2004. "Computational Prediction of Human Metabolic Pathways from the Complete Human Genome." Genome Biology 6 (1): R2. https://doi.org/10.1186/gb-2004-6-1r2.

Steward, J. n.d. "Host Pathways in Coronavirus Replication and COVID-19 Pre-Clinical Drug Target Identification Using Proteomic and Chemoinformatic Analysis." Drug Target Review.

Thiele, I, and B Ø Palsson. 2010. "A Protocol for Generating a High-Quality Genome-Scale Metabolic Reconstruction." Nat Protoc. 5 (1): 93-121.

Thiele, I, N Swainston, RM Fleming, and et al. 2013. "A Community-Driven Global Reconstruction of Human Metabolism." Nat Biotechnol. 31 (5): 419-25. https://doi.org/doi:10.1038/nbt.2488.

Trupp, Miles, Tomer Altman, Carol A Fulcher, Ron Caspi, Markus Krummenacker, Suzanne Paley, and Peter D Karp. 2010. "Beyond the Genome (BTG) Is a (PGDB) Pathway Genome Database: HumanCyc." Genome Biology 11 (Suppl 1): O12-012. https://doi.org/10.1186/gb2010-11-s1-012.

Uzun, Tuğçenur, and Orcun Toptas. 2020. "Artesunate: Could Be an Alternative Drug to Chloroquine in COVID-19 Treatment?" Chinese Medicine 15 (May): 54-54. https://doi.org/10.1186/s13020-020-00336-8.

Wang, H, P Yang, and K Liu. 200AD. "SARS Coronavirus Entry into Host Cells through a Novel Clathrin- and Caveolae-Independent Endocytic Pathway. Cell Res. 2008;18(2):290-301. Doi:10.1038/Cr.2008.15." Cell Res. 18 (2): 290-301.

Wilde, Adriaan H. de, Dirk Jochmans, Clara C. Posthuma, Jessika C. Zevenhoven-Dobbe, Stefan van Nieuwkoop, Theo M. Bestebroer, Bernadette G. van den Hoogen, Johan Neyts, and Eric J. Snijder. 2014. "Screening of an FDA-Approved Compound Library Identifies Four SmallMolecule Inhibitors of Middle East Respiratory Syndrome Coronavirus Replication in Cell Culture." Antimicrobial Agents and Chemotherapy 58 (8): 4875. https://doi.org/10.1128/AAC.03011-14.

$\mathrm{Wu}, \mathrm{Z}$, and JM McGoogan. 2020. "Characteristics of and Important Lessons from the Coronavirus Disease 2019 (COVID-19) Outbreak in China: Summary of a Report of 72314 Cases from the Chinese Center for Disease Control and Prevention. JAMA. 2020 Feb 24." JAMA. https://doi.org/10.1001/jama.2020.2648.

Yan, R, Y Zhang, Y Li, L Xia, Y Guo, and Q Zhou. 2020. "Structural Basis for the Recognition of SARS-CoV-2 by Full-Length Human ACE2. Renhong Yan1,2, Yuanyuan Zhang1,2*, Yaning Li3*, Lu Xia1,2, Yingying Guo1,2, Qiang Zhou1,2†." Science.

Yang, Y., Du, L., Liu, C., Wang, L., Ma, C., Tang, J., Baric, R.S., Jiang, S., and Li, F. (2014). Receptor Usage and Cell Entry of Bat Coronavirus HKU4 Provide Insight into Bat-to-Human Transmission of MERS Coronavirus. Proc. Natl. Acad. Sci. USA 111, 12516-12521. Yang, Y., Liu, C., Du, L., Jiang, S., Shi, Z., Baric, R.S., and Li, F. (2015). Two Mutations Were Critical for Bat-to-Human Transmission of Middle East Respi- Ratory Syndrome Coronavirus. J. Virol. 89, 9119-9123." n.d. 
Yeh, Iwei, Theodor Hanekamp, Sophia Tsoka, Peter D Karp, and Russ B Altman. 2004. "Computational Analysis of Plasmodium Falciparum Metabolism: Organizing Genomic Information to Facilitate Drug Discovery." Genome Research 14 (5): 917-24.

Zhong, Jixin, Jungen Tang, Cong Ye, and Lingli Dong. n.d. "The Immunology of COVID-19: Is Immune Modulation an Option for Treatment?" The Lancet Rheumatology. Accessed June 23, 2020. https://doi.org/10.1016/S2665-9913(20)30120-X.

Zhou, P, and S Yang. 2020. "A Pneumonia Outbreak Associated with a New Coronavirus of Probable Bat Origin." Nature 579: 270-273. 


\section{Figures}

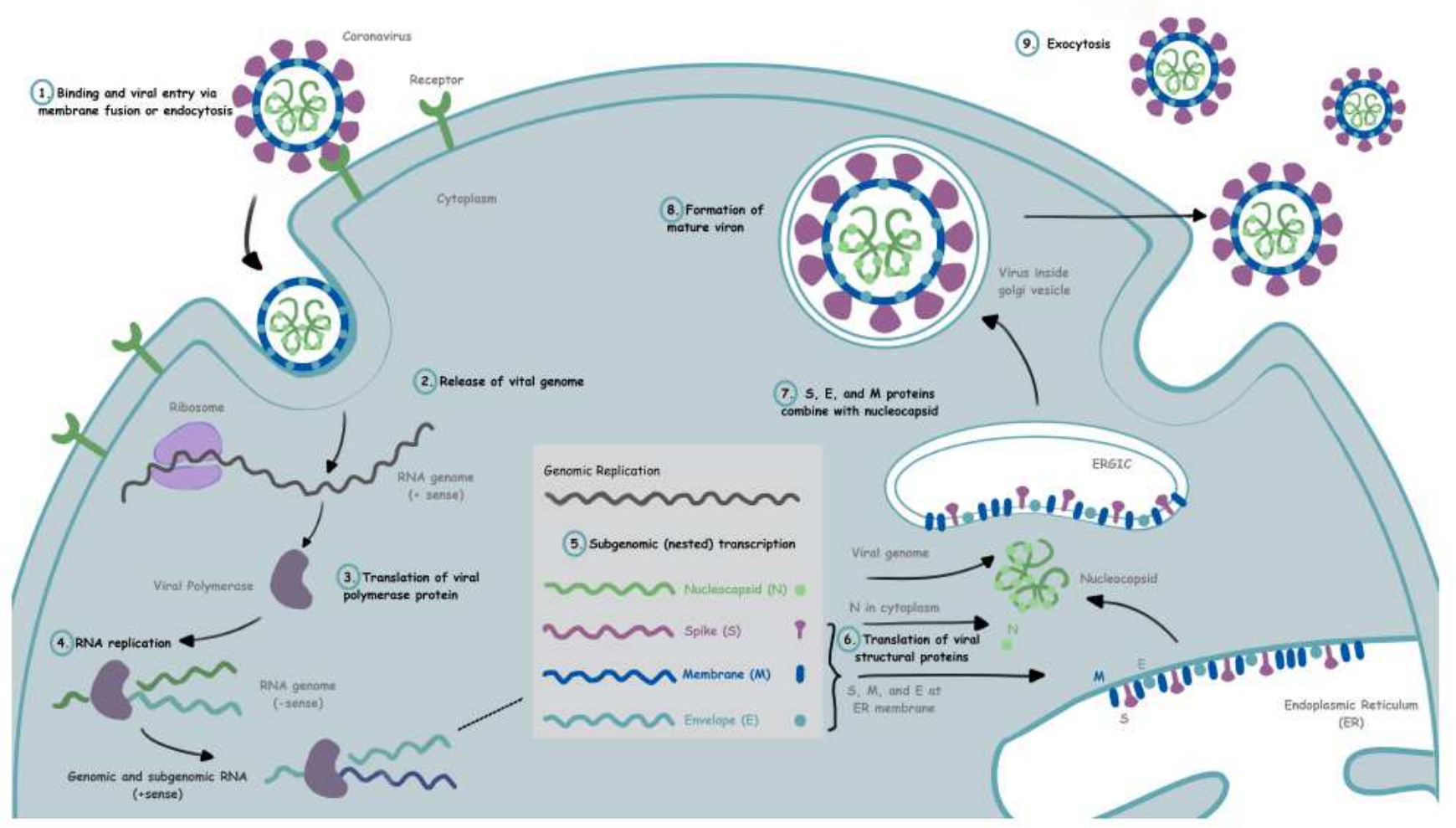

Figure 1

The mechanism of replication of the SARS-CoV-2 virus in the human cell 


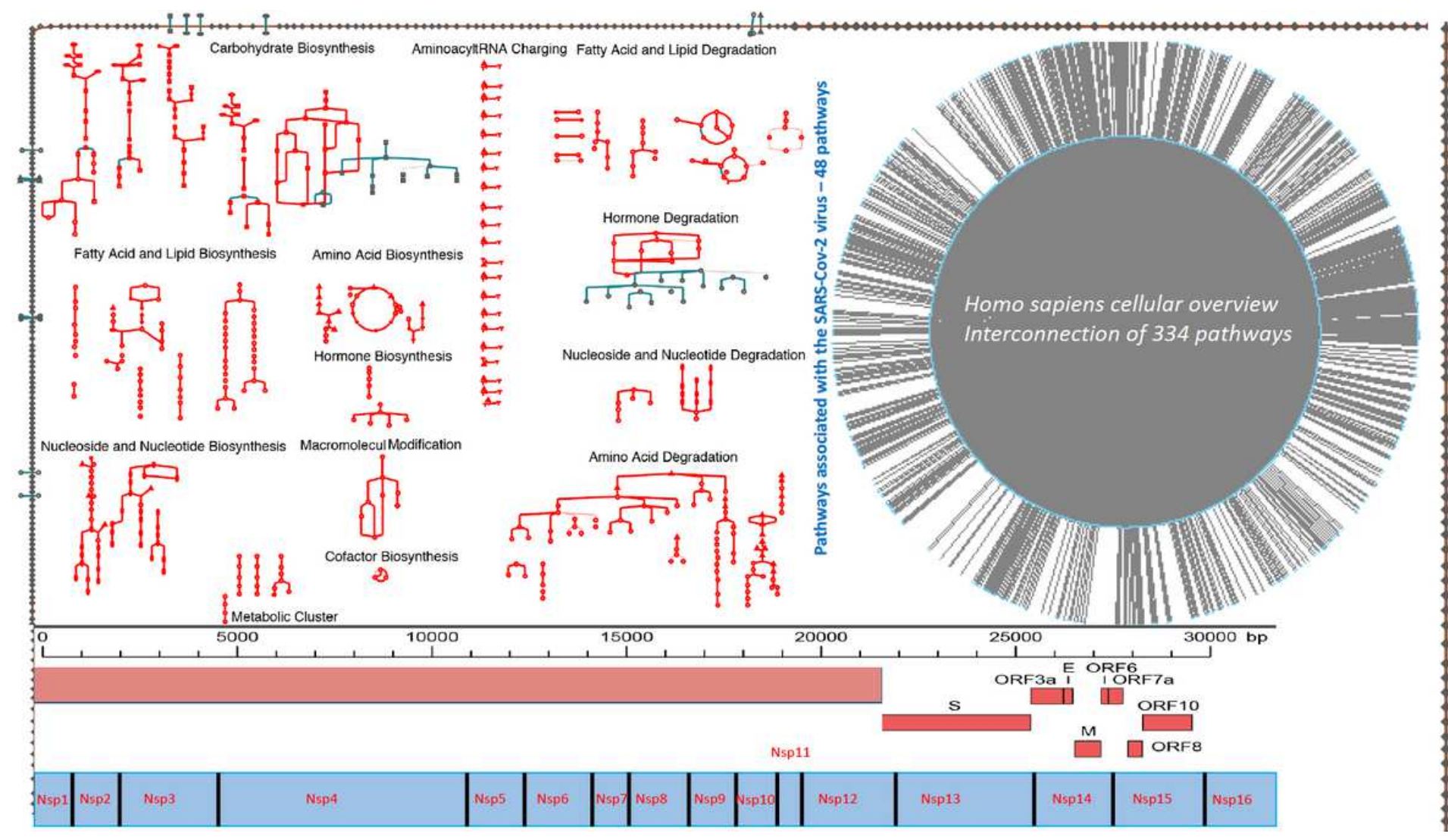

Figure 2

SARS-CoV-2 viral genome \& host dependent metabolic pathways

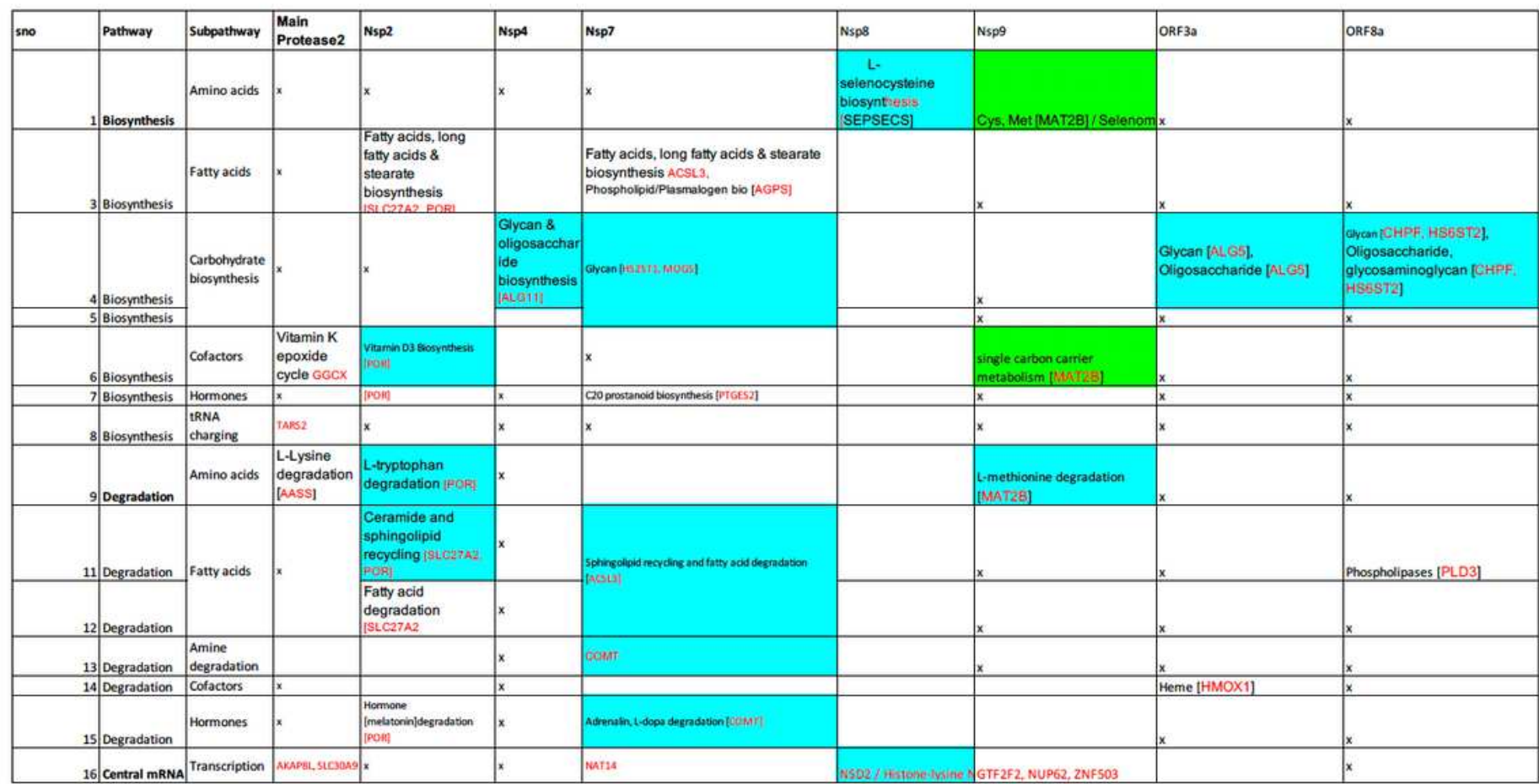


PPi-pathway intersection nodes

\section{Methionine degradation}

A

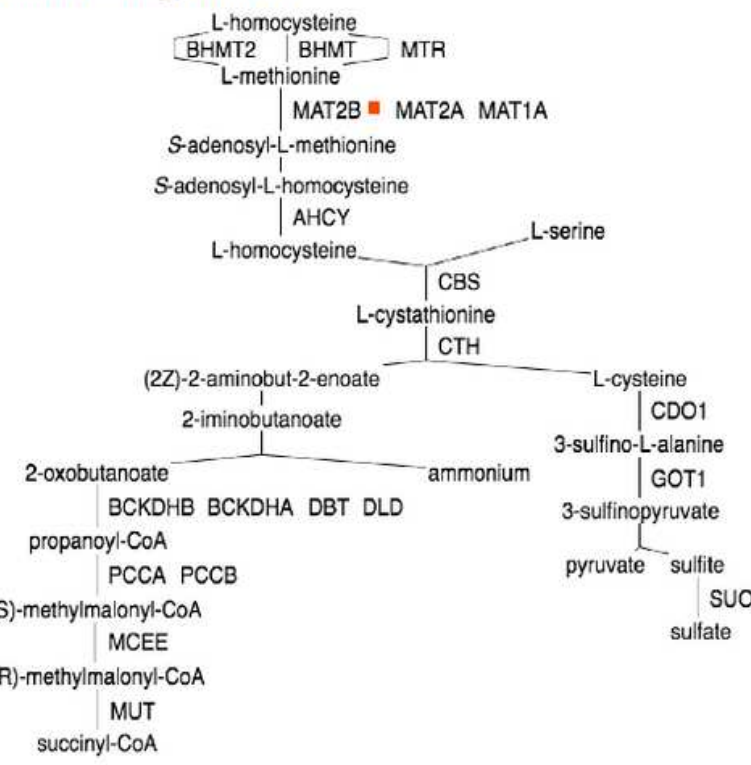

Cysteine Biosynthesis ${ }^{\text {ammonium }}$

S-adenosyl-L-methionine

S-adenosyl-L-homocysteine

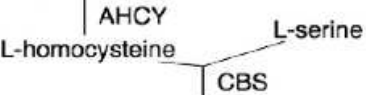

L-cystathionine $\mathrm{CTH}$

(2Z)-2-aminobut-2-enoate L-cysteine

2-iminobutanoate
B

Methionine salvage cycle

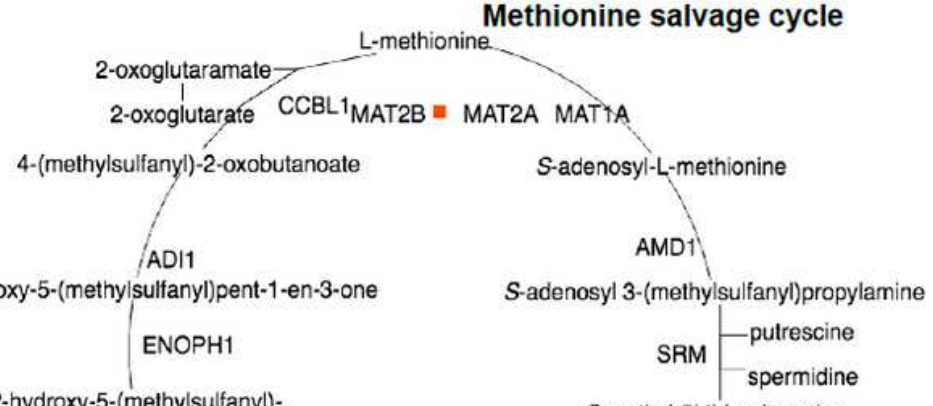

2-hydroxy-5-(methylsulfanyl)-

3-oxopent-1-enyl 1-phosphate

ENOPH1

5-(methylsulfanyl)-2,3-dioxABehtyl 1-phosphate MTAP

S-methyl-5-thio- $\alpha$-D-ribose 1-phosphate

1,2-dihydroxy-5-(methyisulfanyl)pent-1-en-3-one

thyl-5'-thioadenosine
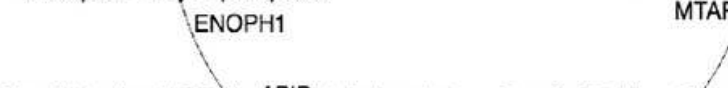

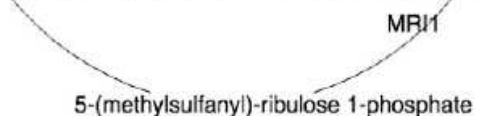

5-(methylsulfanyl)-ribulose 1-phosphate

\section{Figure 4}

PPi-Pathway intersection node - Nsp9 


\section{L-selenocysteine biosynthetic pathway}

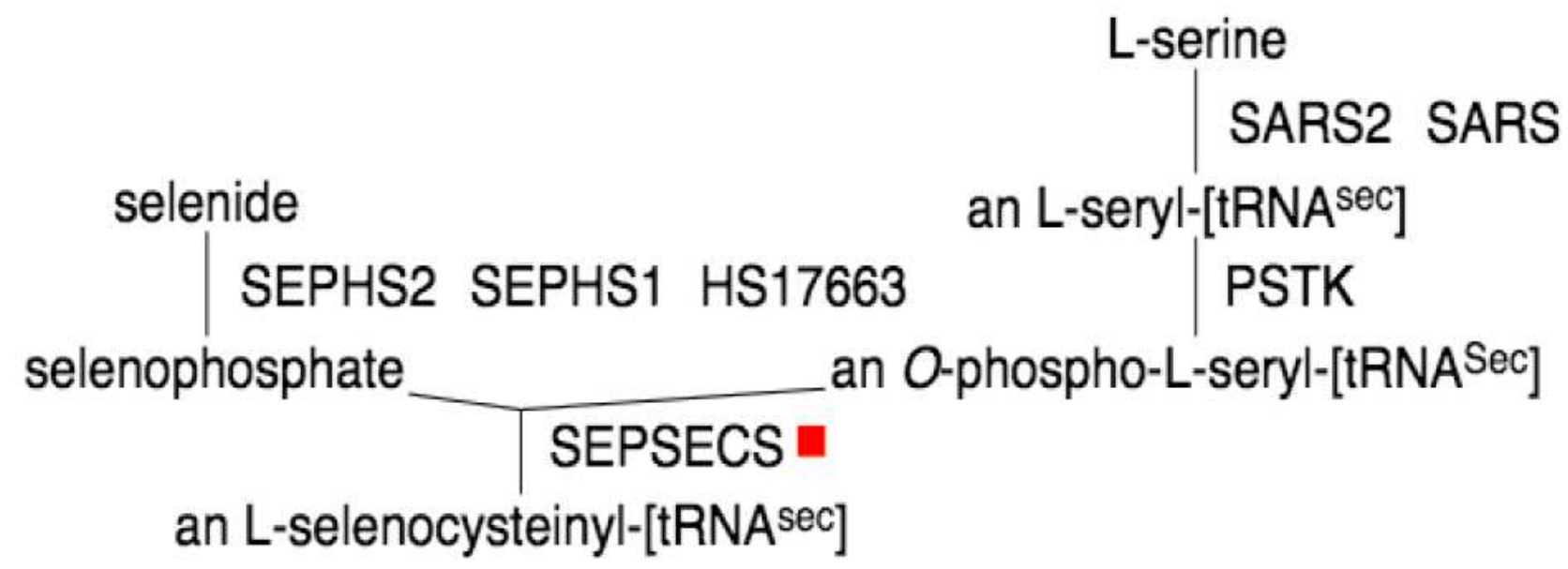

Figure 5

PPi-Pathway intersection node - Nsp8

Fatty acid oxidation

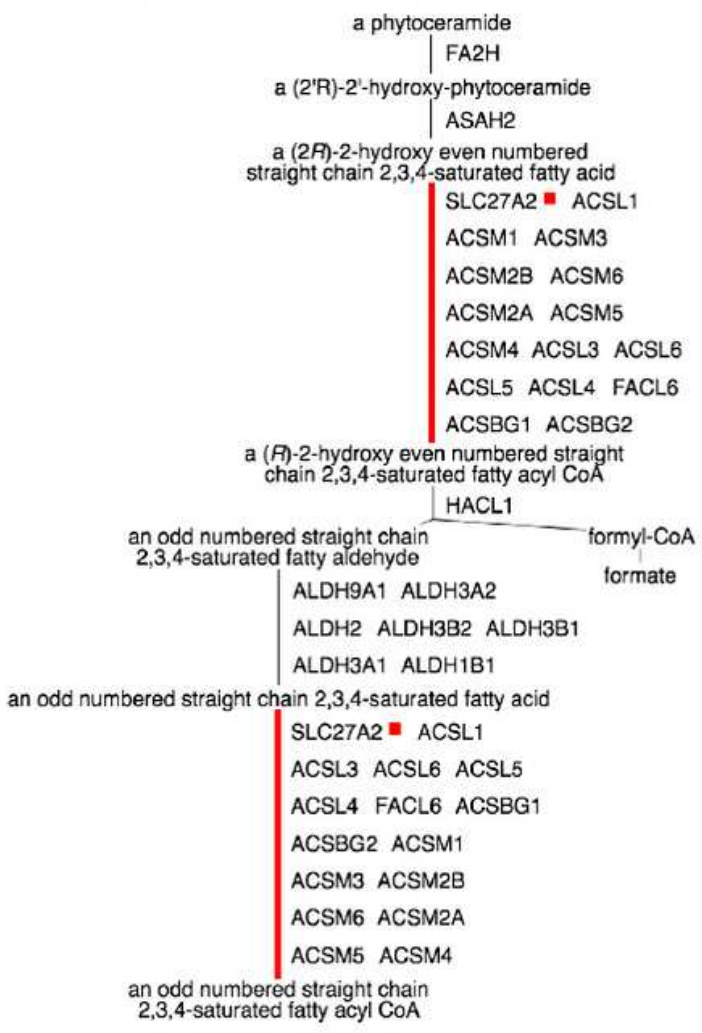

Stearate Biosynthesis

$$
\text { palmitate }
$$

SLC27A2 $=$ ACSL1

FACL6 ACSM1 ACSM3 ACSM2B ACSM6 ACSM2A ACSM5 ACSM4 ACSBG1 ACSBG2 ACSL4 ACSL6 ACSL5 ACSL3 palmitoyl-CoA

ELOVL7 ELOVL6

3-oxooctadecanoyl-CoA

HSD17B12 HADH

(3R)-3-hydroxyoctadecanoyl-COA HSD17B4

(2E)-octadec-2-enoyl-CoA stearoyl-COA ACOT4 ACOT2 OLAH ACOTB ACOT9 ACOT7 stearate

Figure 6 
PPi-Pathway intersection node - Nsp2 as denoted by the red square blocks. The red lines indicate an increase in flux of the highlighted reactions when the virus hijacks the system

\section{N-glycosylation initial phase}

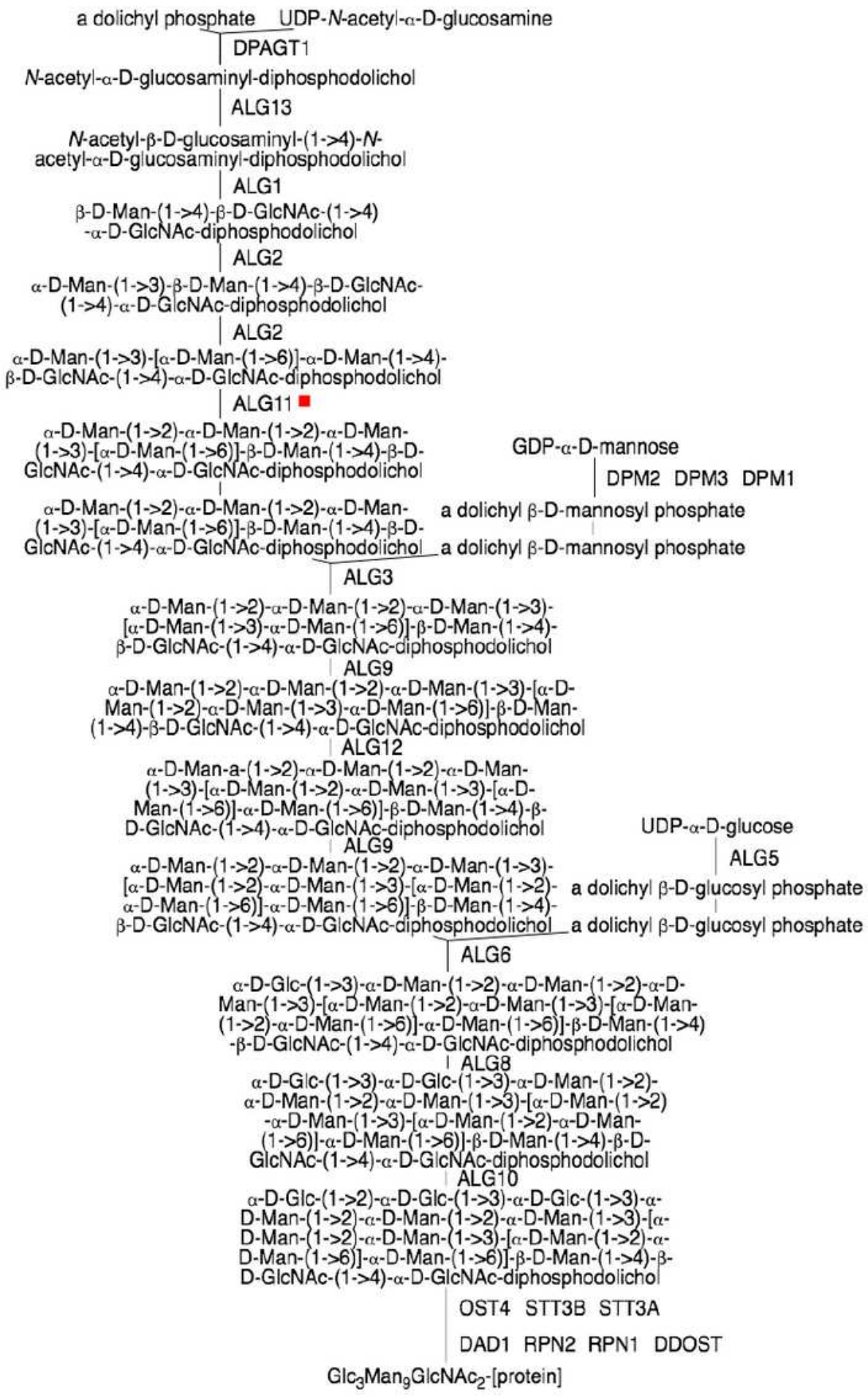

\section{Figure 7}

PPi-Pathway intersection node - Nsp4 


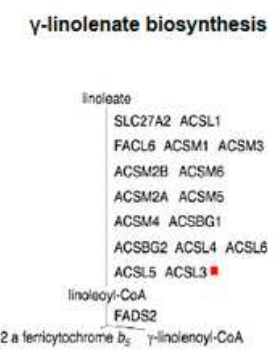

a

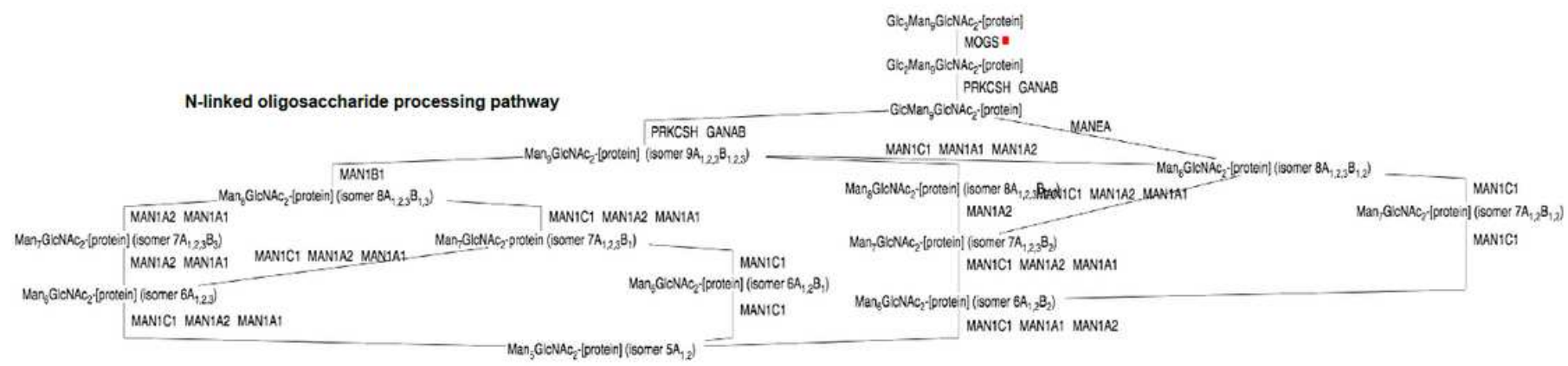

\section{Figure 8}

a. PPi-Pathway intersection node - Nsp7 b. PPi-Pathway intersection node - Orf8a c. PPi-Pathway intersection node - Nsp7 and Orf8a

\section{Supplementary Files}

This is a list of supplementary files associated with this preprint. Click to download.

- Supplementary.zip 